\title{
ARTICLE
}

\section{Neutronic Analysis of Fusion Tokamak Devices by PHITS}

\author{
Atsuhiko M. SUKEGAWA ${ }^{1 *}$, Kouji TAKIYOSHI ${ }^{2}$, Toshio AMANO ${ }^{2}$, \\ Hiromitsu KAWASAKI ${ }^{2}$, and Koichi OKUNO ${ }^{3}$ \\ ${ }^{I}$ Japan Atomic Energy Agency, Naka-shi, Ibaraki 311-0193, Japan \\ ${ }^{2}$ Itochu Techno-Solutions Corporation, Chiyoda-ku, Tokyo 100-6080, Japan \\ ${ }^{3}$ Hazama Technical Research Institute, Tsukuba-shi, Ibaraki 305-0822, Japan
}

\begin{abstract}
A complete 3D neutronic analysis by PHITS (Particle and Heavy Ion Transport code System) has been performed for fusion tokamaku devices such as JT-60U device and JT-60 Superconducting tokamak device (JT-60 Super Advanced). The mono-energetic neutrons $\left(\mathrm{E}_{\mathrm{n}}=2.45 \mathrm{MeV}\right)$ of the $\mathrm{DD}$ fusion devices are used for the neutron source in the analysis. The visual neutron flux distribution for the estimation of the port streaming and the dose rate around the fusion tokamak devices has been calculated by the PHITS. The PHITS analysis makes it clear that the effect of the port streaming of superconducting fusion tokamak device with the cryostat is crucial and the calculated neutron spectrum results by PHITS agree with the MCNP-4C2 results.
\end{abstract}

\section{KEYWORDS: Neutronic Analysis, Fusion Tokamak Device, JT-60U, JT-60SA, PHITS, MCNP}

\section{Introduction}

The conceptual design of fusion tokamak device has been performed by neutoronic analysis ${ }^{1}$ using one-dimensional and two-dimensional discrete ordinate codes (ANISN ${ }^{2)}$ and DOT $\left.3.5^{3)}\right)$. Recently, the general trend of shielding calculation of fusion device, tokamak, is to use three-dimensional (3D) codes.

PHITS (Particle and heavy Ion Transport code System) ${ }^{4)}$ is a 3D Monte Calro transport code system for all particles with including neutron, photon, proton, hadron, nucleus, electron and Heavy ions up to $200 \mathrm{GeV}$. The system is able to deal with lower than $20 \mathrm{MeV}$ neutron and low energy photon and electron transport based on evaluated nuclear data library with the same model as MCNP-4C code ${ }^{5)}$.

In a fusion tokamak device, high performance plasmas using deuterium by JT-60U device had been developed from 1991 to 2008 . The total neutron yields were $1.52 \times 10^{20} \mathrm{n}$. It is necessary to assess the radiation duty assignments for the disassembly of JT-60 device. So, we need to calculate the neutron spectrum in the structure materials of the JT-60U device using the $3 \mathrm{D}$ codes for the activation analysis.

In addition, an advanced deuterium-deuterium (DD) fusion device as Superconducting fusion tokamak device: JT-60SA (JT-60 Super Advanced) will be constructed in the existing JT-60 facilities and be operated for more than ten years with DD discharges. The annual neutron emission from the steady state plasma will increase by about fifty times the permitted amount of neutron emission in the JT-60U device. Hence, the neutron emitted by JT-60SA must be shielded more effectively in the vacuum vessel, the cryostat and the JT-60 building.

In particular, it is necessary to improve the neutron shielding performance by the vacuum vessel of JT-60SA. For JT-60SA, however, the water layer thickness of vacuum vessel is limited to $135 \sim 150 \mathrm{~mm}$ as specified from the size of TF superconducting coil in which $\mathrm{NbTi}$ winding is newly used. The radiation shielding performance of the vacuum vessel by using pure water was insufficient, so that we selected the water with boric acid, namely borated water, instead of pure water. The thickness of the borated water layer $140 \mathrm{~mm}$ was adopted from the radiation shielding design of toroidal field magnetic (TF) superconducting winding by $1 \mathrm{D}$ calculation ${ }^{1)}$.

In the present radiation shielding design, the nuclear responses such as the neutron flux distribution, the photon flux distribution, the nuclear heating of the superconducting coils, the duct streaming on the facility and the dose rate around the device have been calculated by 3D discrete ordinate method $^{6}$ and 3D Monte Carlo method ${ }^{7}$.

Therefore, to improve the accuracy of the radiation shielding design with the $3 \mathrm{D}$ visual neutron and photon transport analysis such as the PHITS calculation for the radiation safety of the facility is demanded.

In this paper, we discuss the availability and the advantage of PHITS for neutronic analysis of fusion tokamak devices such as JT-60U and the JT-60SA.

\section{Radiation Shielding}

For the radiation safety on fusion tokamak devices, it is important to resolve the nuclear responses into the following points: (1) estimation of biological shielding during the operation; (2) estimation of the dose rate by the activation in areas required to access after the operation; (3) the safety operation of the superconducting coil during the operation.

In JT-60U fusion tokamak device, neutrons by the DD fusion reaction in the plasmas are emitted from the vacuum vessel. The NBI (Neutral Beam Injector) and RF (Radio Frequency) heating system and the diagnostic system are arranged in surroundings of the vacuum vessel. As a neutron shielding method of JT-60U device for the radiation safety, a large-scale shielding structure by the JT-60 building was adopted including the area away from the vacuum 
vessel.

The JT-60 building is shielded by the $2000 \mathrm{~mm}$ thick ordinary concrete on the ceiling by the shielding materials composed on the ceiling of concrete panels of $500 \mathrm{~mm}$, polyethylene of $150 \mathrm{~mm}$ and the concrete of $150 \mathrm{~mm}$ thicknesses.

Table 1 Neutron emission rates of JT-60U and JT-60SA

\begin{tabular}{cccr}
\hline Neutron Emission Rates & $\begin{array}{c}\text { JT-60SA } \\
\text { Expected }\end{array}$ & $\begin{array}{c}\text { JT-60U } \\
\text { Permitted }\end{array}$ & JT-60SA/JT-60U \\
\hline $\mathrm{n} / \mathrm{sec}$ & $4.0 \times 10^{17}$ & $2.0 \times 10^{17}$ & 2.0 \\
$\mathrm{n} /$ week & $2.5 \times 10^{20}$ & $3.1 \times 10^{18}$ & 80.6 \\
$\mathrm{n} / 3$ months & $1.0 \times 10^{21}$ & $2.1 \times 10^{19}$ & 47.6 \\
$\mathrm{n} /$ year & $1.5 \times 10^{21}$ & $3.1 \times 10^{19}$ & 48.4 \\
\hline
\end{tabular}

Table 1 shows the neutron emission rates of JT-60U and JT-60SA. The neutron emission rates from the JT-60SA plasma will be planned to increase the permitted rates in the JT-60U. The improvement of the shielding performance of JT-60SA are entrusted by mainly the vacuum vessel and the cryostat as an additional shielding for the compact shielding concept due to the restricted space in the building.

The structure of the vacuum vessel and the cryostat is possible to be simplified. For safety operation of the superconducting TF coils, the vacuum vessel is required to suppress the nuclear heating at the TF coil.

At first, the vacuum vessel of the JT-60SA was designed by the double-wall structure with pure water to shield the neutrons. To improve the neutron shielding performance, the solubility to the pure water of the boric acid hit on a function of the water temperature. The solubility is $8.2 \mathrm{wt} \%$ at $40^{\circ} \mathrm{C}$ on the water temperature. In addition, the concentration ratio of ${ }^{10} \mathrm{~B}$ ( $20 \%$ in nature) in the borated water is enriched up to $95 \%$. Consideration of the shielding effect of the cryostat for biological shielding in the torus hall, the borated water as the shielding material is adopted to initial JT-60SA shielding design. The structure consists of double wall using the 24 $\mathrm{mm}$ thickness of SS316L filled with the $140 \mathrm{~mm}$ thick borated water ${ }^{1)}$.

In the basic structure design of the cryostat for the demanded neutron yields $\left(1.5 \times 10^{21} \mathrm{n} /\right.$ year as the maximum $)$, the dose rate at the surface of the cryostat was decided to secure the acceptance for maintenance using a novel boron-loaded low activation concrete. The cryostat consists of the $220 \mathrm{~mm}$ thickness of the concrete between two SS304 panels of $34 \mathrm{~mm}$ (inboard) and $6 \mathrm{~mm}$ (outboard) thicknesses. The low activation concrete contaminated with $\mathrm{B}_{4} \mathrm{C}$ (Boron: $2 \mathrm{wt} \%$ ) is adopted to improve the shielding efficiency of the thermal neutrons through the vacuum vessel ${ }^{8)}$.

\section{Calculation}

Three-dimensional radiation shielding analysis has been carried out in order to assess the detailed information on radiation environment of JT-60U and JT-60SA in the JT-60 building. The calculations have been carried out using $3 \mathrm{D}$
Monte Carlo code PHITS-2.13 with JENDL-3.3 $3^{9-10)}$ as nuclear data library. The PHITS has been improved for the analysis of the tokamak fusion device. The mono-energetic neutrons $\left(E_{n}=2.45 \mathrm{MeV}\right)$ of the DD fusion devices are used for the neutron source in the analysis. The neutron source distribution is a toroidally symmetric source for the analysis. The toroidal source (S) has a poloidal distribution such as Eq. 1, so that a source program in PHITS has been replaced by a newly developed source program.

$$
\mathrm{S}=\left[1-\left\{\frac{\left(\mathrm{R}-\mathrm{R}_{\mathrm{p}}\right)^{2}}{\mathrm{a}_{\mathrm{p}}^{2}}+\frac{\left(\mathrm{Z}-\mathrm{Z}_{\mathrm{p}}\right)^{2}}{\mathrm{a}_{\mathrm{p}}^{2} \kappa^{2}}\right\}\right]^{\mathrm{m}}
$$

where $R_{p}$ is the major radius, $a_{p}$ is the minor radius, $Z_{p}$ is the vertical shift of the plasma center, $\kappa$ is the ellipticity ${ }^{11)}$. In addition, we considered the plasma triangularity in the expressions. Table 2 shows the plasma parameters of JT-60U and JT-60SA for the analysis

Table 2 Plasma parameters of JT-60U and JT-60SA

\begin{tabular}{rrr}
\hline & \multicolumn{1}{c}{ JT-60U } & \multicolumn{1}{c}{ JT-60SA } \\
\hline $\mathrm{R}_{\mathrm{p}}$ & 347.00 & 303.15 \\
$\mathrm{a}_{\mathrm{p}}$ & 98.00 & 115.15 \\
$\mathrm{Z}_{\mathrm{p}}$ & 17.00 & 0.00 \\
$\mathrm{\kappa}$ & 1.40 & 1.91 \\
$\mathrm{~m}$ & 2.00 & 2.00 \\
\hline
\end{tabular}

The visual nuclear responses such as the neutron flux distribution and the photon flux distribution for the estimations of the nuclear heating of the coils and the dose rate around the devices have been calculated by PHITS for the fusion tokamak devices.

The bird's-eye view made by the input data for the analysis of JT-60U device is shown in Figure 1.

The model of the device have been developed describing a 360 degree sector. In JT-60SA model, we have been made use of a 360 degree sector on the calculation as well as in the JT-60U device.

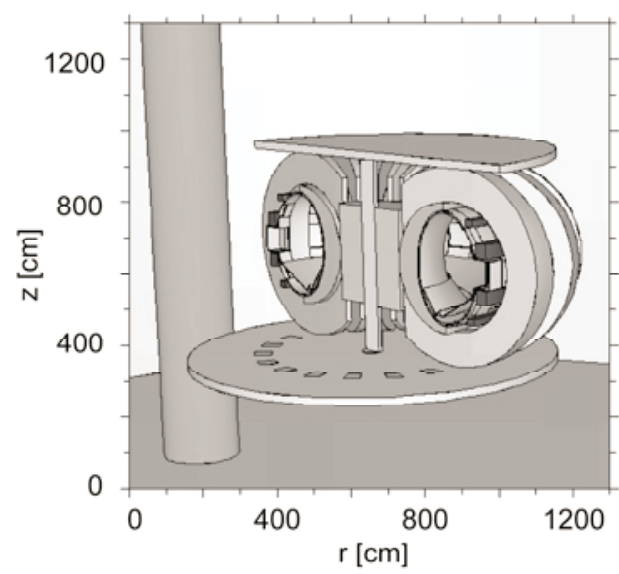

Fig.1 3D model plot of JT-60U by ANGEL

\section{Results and Discussion}




\section{Neutron Flux Distribution}

In comparison of the total neutron flux distributions on JT-60U and JT-60SA, the cross sections of JT-60U and JT-60SA device in the torus hall are shown in Figure 2.

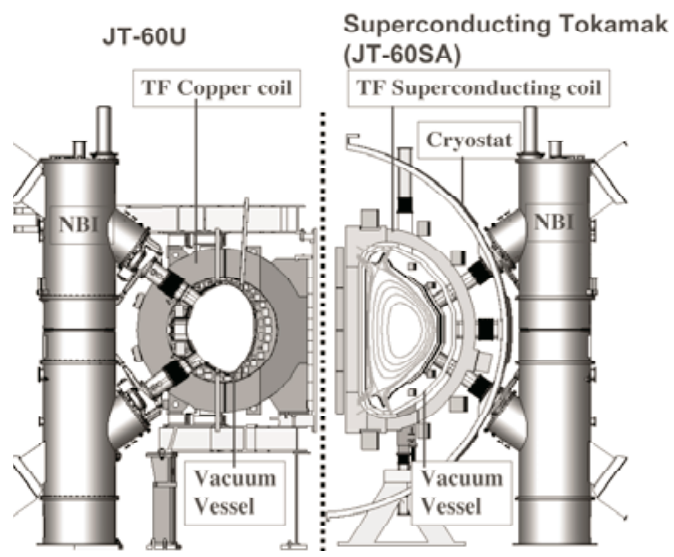

Fig.2 Cross section of JT-60U and JT-60SA

From the poroidal view of the calculation results, the total neutron distributions described at 2D (R-Z) plane around the JT-60U and JT-60SA devices by PHITS analysis are shown in Figure 3.

In comparison of the total neutron flux distributions on JT-60U and JT-60SA, the additional shielding does not make use around the vacuum vessel of JT-60U. The PHITS analysis makes it clear that the neutron shielding performance of JT-60SA is improved by the vacuum vessel and the cryostat.

The total neutron distributions described at 2D (R-Z) plane in JT-60 torus hall by PHITS analysis are shown in Figure 4 with JT-60U and the JT-60SA. The neutron leakage from the three ports at the cryostat was vividly analyzed on the JT-60SA. The phenomenon such as Ray Effect on the port streaming analysis using discrete ordinate methods was not observed as a characteristic on the PHITS analysis. In the JT-60SA, the influence of the port streaming is improved at about $15 \mathrm{~m}$ of r-direction and $10 \mathrm{~m}$ of the $\mathrm{z}$-direction though the total neutron flux level of $10^{-9}$ on the devices peripheral is about one order lower than that of $10^{-8}$ in the surrounding of the JT-60 building.

From the toroidal view (: mid-plane cross section) of the calculation results, the total neutron distribution at $2 \mathrm{D}(\mathrm{X}-\mathrm{Y})$ mid-plane around the vacuum vessel is shown in Figure 5. The neutron shielding performance at the TF coil of JT-60SA is improved by the vacuum vessel.

The total neutron distribution at 2D (X-Y) mid-plane in the torus hall is shown in Figure 6. From the analysis, it was clarified that the effects of neutrons by the port streaming of the JT-60SA are clearly depicted in JT-60 torus hall. So, the radiation shielding conceptual design of superconducting tokamak device such as JT-60SA not only consider the structure of the vacuum vessel and the cryostat but also have to assess the effect of the port streaming by $3 \mathrm{D}$ codes.

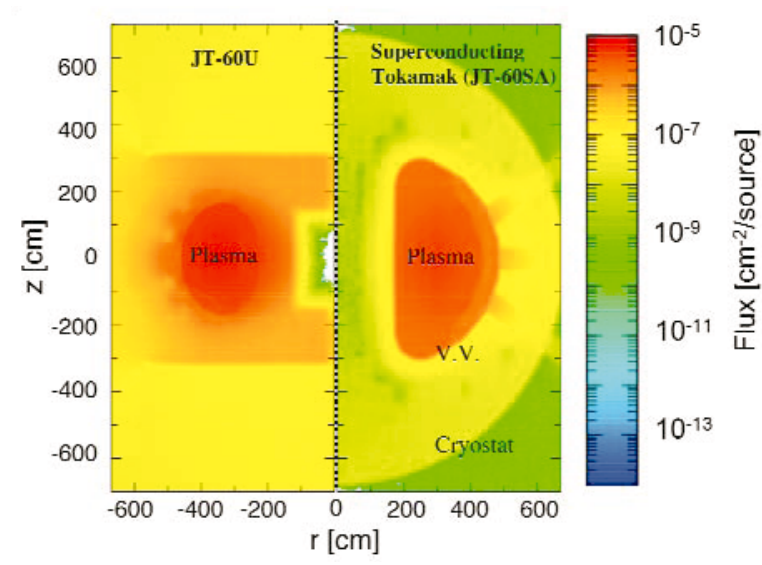

Fig.3 Poloidal view of total neutron distributions around JT-60U and JT-60SA

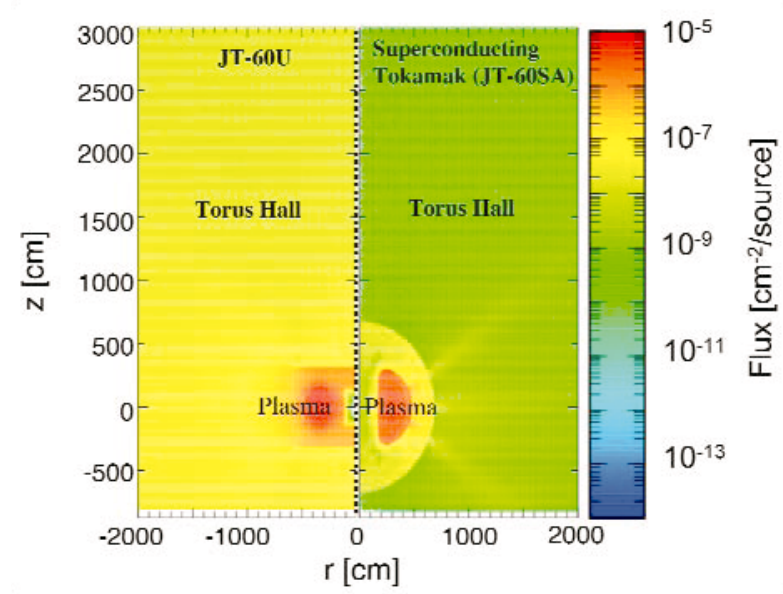

Fig. 4 Poloidal view of total neutron distributions of JT-60U and JT-60SA in JT-60 torus hall

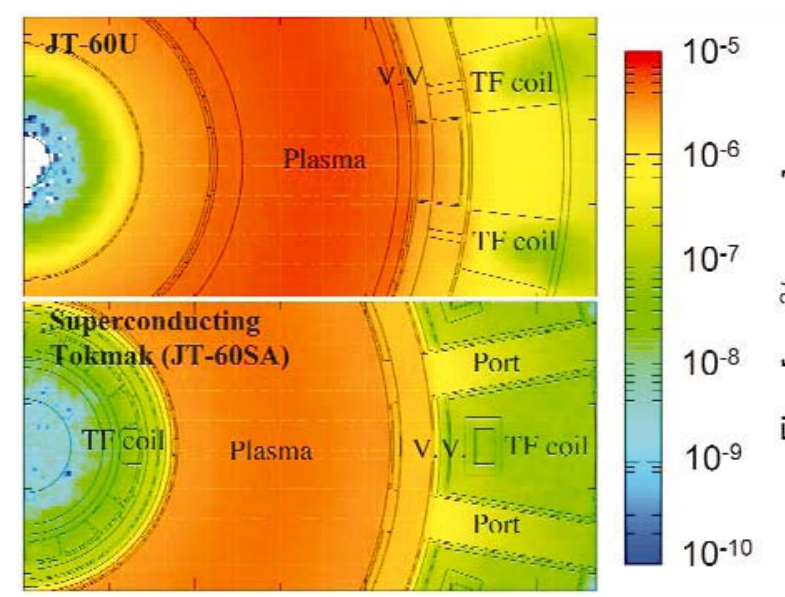

Fig. 5 Toroidal view of total neutron distributions around the vacuum vessel of JT-60U and JT-60SA 


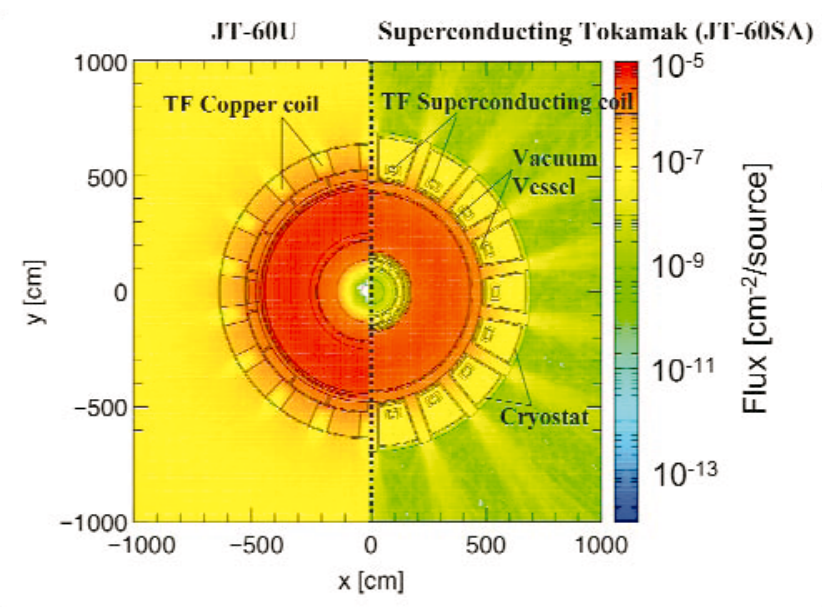

Fig. 6 Toroidal view of total neutron distributions of JT-60U and JT-60SA in JT-60 torus hall

\section{Neutron Spectrum}

To assess the influence on neutron transport by the PHITS and the MCNP-4C2, the neutron spectra of various points during DD operation in the JT-60U and the JT-60SA have been evaluated by the track-length tallies. The neutron spectrums at TF coil conduit on the JT-60SA by the PHITS analysis and MCNP-4C2 analysis are shown in Figure 7. The line and dot-line indicate the calculated results for the PHITS and MCNP-4C2, respectively. The PHITS results agree with the MCNP-4C2 results.

The advantage of the PHITS analysis different from the MCNP-4C2 analysis is able to obtain the peculiar results by the visualization. However, the MCNP-4C2 does not have the mesh tally function of MCNP- $5^{12)}$. So, it is necessary to evaluate the distributional results of the PHITS by comparing it with the mesh tally of the MCNP-5.

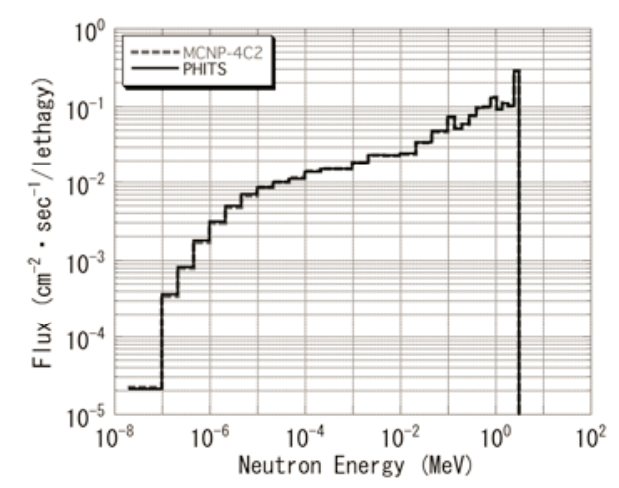

Fig. 7 Neutron spectrums at TF coil conduit by PHITS and MCNP-4C2

\section{Conclusion}

A complete $3 \mathrm{D}$ radiation shielding analysis by PHITS has been performed for the Fusion Tokamak Devices.

It is possible to make use of PHITS in the neutronic analysis of the devices by improving the source routine for the tokamak devices. The neutron transport analysis by PHITS makes it clear that the shielding performance of the superconducting fusion tokamak device (JT-60SA) with the cryostat is improved by vacuum vessel and cryostat by the graphical results of the PHITS, and the effect of the port streaming of superconducting fusion tokamak device such as the JT-60SA with the cryostat is crucial. From the standpoint of the port streaming, it is necessary to calculate by $3 \mathrm{D}$ codes for the neutronic analysis of the fusion tokamak devices.

The calculated neutron spectrum results by PHITS agree with the MCNP-4C2 results at various estimation points with the track length tally on the JT-60U device and JT-60SA device.

In the near future, the $3 \mathrm{D}$ neutronic analysis of the additional heating system such as the NBI and the RF of the JT-60SA for the maintenance will be performed by the PHITS. In addition, the neutronic analysis of the JT- 60 building with the JT-60SA by Event Generator Mode of the PHITS will be planned.

\section{Acknowledgments}

The authors would like to thank Dr. K. Niita for this contribution. We would like to acknowledge Dr. N. Miya and Dr. T. Nishitani for their kind suggestions.

\section{References}

1) A. M. Sukegawa, S. Sakurai, et al, "Safety design of radiation shielding for JT-60SA", Fus. Eng. Des. 82 , 2799 (2007).

2) Engle, W. A. Jr., “A User's Manual for ANISN, A One-dimensional Discrete Ordinates Transport Code with anisotropic Scattering”, K-1693 (1967).

3) Rhoades, W. A., et al.,DOT3.5 Two-Dimensional Discrete Ordinates Radiation Transport Code, CCC-276 (1977).

4) H. Iwase, K. Niita, T. Nakamura, Development of General-Purpose Particle and Heavy Ion Transport Monte Carlo Code, J. Nucl. Sci. Technol., 39, 1142 (2002)

5) Judith F. Briesmeister., MCNP - A General Monte Carlo N-Particle Transport Code Version 4C, LA-13709-M (2000)

6) Radiation Transport Software: ATTILA, Transpire Inc. (2006) : www.trancepireinc.com

7) R. Villari et al," Neutronic analysis of the JT-60SA toroidal magnets", Fus. Eng. Design. 84, 1947 (2009)

8) A. Morioka et al, "Irradiation and penetration tests of boron-doped low activation concrete using $2.45 \mathrm{MeV}$ and $14 \mathrm{MeV}$ neutron sources", J. Nucl. Mater. 329-333, 1619(2004).

9) K. Shibata et al.," Japanese Evaluated Nuclear Data Library,Version-3 - JENDL-3.3", J. Nucl. Sci. Technol. 39[11], 1125-1136 (2002).

10) K. Kosako et al., "The Libraries FSXLIB and MATXSLIB Based on JENDL-3.3", JAERI-Data/Code 2003-011, Japan Atomic Energy Research Institute (2003).

11) T. Nishitani et al.,"Absolute calbration of the JT-60U neutron monitors using a ${ }^{252} \mathrm{Cf}$ neutron source", Rev. Sci. Instrum. 63[11], 5270 (1992).

12) X-5 Monte Carlo Team, MCNP-A General Monte Carlo N-Particle Trasport Code, Version 5, LA-UR-03-1987 Los Alamos National Laboratory (2003) 